\title{
Generalized Dirac monopoles in non-Abelian Kaluza-Klein theories
}

\author{
Ion I. Cotăescu * \\ West University of Timişoara, \\ V. Pârvan Ave. 4, RO-300223 Timişoara, Romania
}

\begin{abstract}
A method is proposed for generalizing the Euclidean Taub-NUT space, regarded as the appropriate background of the Dirac magnetic monopole, to non-Abelian Kaluza-Klein theories involving potentials of generalized monopoles. Usual geometrical methods combined with a recent theory of the induced representations governing the TaubNUT isometries lead to a general conjecture where the potentials of the generalized monopoles of any dimensions can be defined in the base manifolds of suitable principal fiber bundles. Moreover, in this way one finds that apart from the monopole models which are of a space-like type, there exists a new type of time-like models that can not be interpreted as monopoles. The space-like models are studied pointing out that the monopole fields strength are particular solutions the Yang-Mills equations with central symmetry producing the standard flux of $4 \pi$ through the two-dimensional spheres surrounding the monopole. Examples are given of manifolds with Einstein metrics carrying $S U(2)$ monopoles.
\end{abstract}

Pacs 04. 62. $+\mathrm{v}$

Key words: Kaluza-Klein, Yang-Mills, monopole, induced representation

*E-mail: cota@physics. uvt.ro 


\section{Introduction}

A special class of solutions of the Maxwell or Yang-Mills equations are the instantons and monopoles defined on appropriate flat or curved backgrounds [1, 2. Successful methods were used for investigating the geometric properties of the original Dirac magnetic monopole [3] and its Abelian [4] or nonAbelian generalizations [5, 6, 7]. Moreover, the study of the role of the BPS monopoles [8] in the Lagrangian theories with Higgs mechanisms is actually of a large interest [9].

Another framework is offered by the Kaluza-Klein theories where the Maxwell or Yang-Mills degrees of freedom deal with specific extra-coordinates exceeding the physical spacetime. In these theories the basic problem is to find solutions of the Yang-Mills equations in a geometry whose global metric should be an exact solution of the Einstein equations [10. A typical example is the four-dimensional Euclidean Taub-NUT space which involves the potentials of the Dirac magnetic monopole and satisfies the vacuum Einstein equations. Moreover, this geometry is hiper-Kähler having many interesting properties related to a specific hidden symmetry 11. For this reason the Kählerian geometries were considered for generalizing the Dirac monopole to many dimensions [4].

In this article we should like to continue the investigation of the geometrical methods that could lead to new versions of generalized Dirac monopoles in non-Abelian Kaluza-Klein theories of any dimensions. In this matter there are different opinions concerning the possible topologies of the backgrounds carrying generalized monopoles [12, 9]. Here we start with the idea that the non-Abelian monopoles may have similar topologies and the same type of invariants as the Abelian Dirac one. We assume that the monopole field is (I) a particular solution of the Yang-Mills equations with an apparent string singularity that (II) can be reduced up to a point-like singularity grace to a suitable topology [2]. This remaining singularity represents the monopole which must give rise to a field strength with (III) a central symmetry up to gauge transformations and (IV) producing through two-dimensional spheres surrounding the monopole the standard flux of $4 \pi$, in units of the coupling constant [5]. In order to accomplish these requirements we shall focus on the relation between the geometrical and the gauge symmetries [13, 10].

In a recent study concerning the isometries of the Euclidean Taub-NUT space [14] we observed that the angular coordinates of the four-dimensional Taub-NUT space form a set of parameters of the isometry group of the phys- 
ical three-dimensional subspace. This property explains why the isometries of the Euclidean Taub-NUT space involve linear rotations of the Cartesian physical coordinates while the extra-coordinate transforms according to a non-linear representation of the rotation group. We have found the integral form of this representation showing that this is induced by the subgroup of the rotations that preserve the direction of the string giving rise to the Dirac monopole.

Here we intend to generalize this conjecture combining our method of induced representations [14] with the usual theory of the principal fiber bundles. We consider that the manifolds of our non-Abelian Kaluza-Klein theories are principal bundles where the fibers are orthogonal groups and the base manifolds are physical spaces with a manifest central symmetry. In this context we propose the concrete integral form of the isometry transformations and derive the representations induced by the structure group of the principal bundle in the general case of any (pseudo)-orthogonal isometry group. Hereby we deduce the gauge transformations associated to isometries that guarantee the central symmetry of the entire theory and offer us the tools for eliminating the original string singularity within a suitable non-trivial fibration [2, 7, 15. The remaining monopole singularity gives rise to fields strength with central symmetry that satisfy the sourceless Yang-Mills equations. Moreover, we show that these solutions are topologically stable in the sense that their principal bundles are non-trivial only over a two-dimensional sphere surrounding the monopole 9]. Then it is natural to find that the principal invariant of these models is just the desired standard flux.

We shall achieve these general objectives, starting in the second section with a short presentation of the lesson offered by the Euclidean Taub-NUT geometry that will guide us to a natural generalization of the monopole geometry. The next section is devoted to some technical details concerning the method of introducing coordinates in orbits related to the Lie groups and involved in the fiberings we use. In addition, the horizontal and vertical projections in the tangent spaces of the principal bundles are also considered. Our main results are obtained in the fourth section where we introduce the isometries of our approach which generate the gauge transformations we need for giving up the effects of the string singularities without to affect the global central symmetry. Moreover, we show that beside the usual monopole models having the strings in a space-like direction, there exists another type of models with time-like strings but correct unitary gauge groups. However, since these models are rather unusual, we analyze in the fifth section only the 
space-like models obtaining the general solutions for the potentials and the corresponding fields strength. We show how can be eliminated the string singularity and study the topological stability of the monopole calculating the flux of its field strength. Examples of $S U(2)$ monopoles in concrete Einstein spaces are given before to present the final comments.

We use the natural units with $\hbar=c=1$ and consider unit coupling constants.

\section{The lesson of the Taub-NUT geometry}

The Euclidean Taub-NUT manifold is the space of the Abelian Kaluza-Klein theory of the Dirac magnetic monopole that provides a non-trivial generalization of the Kepler problem. This space is a special member of the family of four-dimensional manifolds, $M_{4} \sim \mathbb{R}^{4}$, equipped with the isometry group $\mathbf{I}\left(M_{4}\right)=S O(3) \otimes U(1)$ and carrying the the Dirac magnetic monopole related to a string along the third axis.

These geometries can be easily constructed in local charts with spherical coordinates $(r, \theta, \varphi, \alpha)$ among them the first three are the usual spherical coordinates of the vector $\vec{x}=\left(x^{1}, x^{2}, x^{3}\right)$, with $|\vec{x}|=r$, while $\alpha$ is the Kaluza-Klein extra-coordinate of this chart. The spherical coordinates can be associated with the Cartesian ones $(x, y)=\left(x^{1}, x^{2}, x^{3}, y\right)$ where the extracoordinate is up to a factor $y=-(\alpha+\varphi)$. In Cartesian coordinates one has the opportunity to use the vector notation and the scalar products $\vec{x} \cdot \vec{x}^{\prime}$ which are invariant under the $S O(3)$ rotations.

The group $S O(3) \subset \mathbf{I}\left(M_{4}\right)$ has three independent one-parameter subgroups, $S O_{i}(2), i=1,2,3$, each one including rotations $R_{i}(\psi)$, of angles $\psi \in[0,2 \pi)$ around the axis $i$. With this notation any rotation $R \in S O(3)$ in the usual Euler parametrization reads $R(\alpha, \beta, \gamma)=R_{3}(\alpha) R_{2}(\beta) R_{3}(\gamma)$. Moreover, we can write $\vec{x}=R(\varphi, \theta, 0) \vec{x}_{o}$ where the vector $\vec{x}_{o}=(0,0, r)$ along the string direction is invariant under the rotations $R_{3} \in S O_{3}(2)$. For this reason one says that $\mathrm{SO}_{3}(2)$ is the little group of the string direction $\vec{x}_{o}$. This group will be the second term of the isometry group $\mathbf{I}\left(M_{4}\right)$ usually denoted by $U(1)$ since these two groups are isomorphic.

We have shown [14] that the transformations of the isometry group $\mathbf{I}\left(M_{4}\right)$ can be written explicitly in an integral form defining the action of two arbitrary rotations, $R \in S O(3)$ and $R_{3} \in S O_{3}(2) \sim U(1)$, in the spherical charts, $\left(R, R_{3}\right):(r, \theta, \varphi, \alpha) \rightarrow\left(r, \theta^{\prime}, \varphi^{\prime}, \alpha^{\prime}\right)$, such that $R\left(\varphi^{\prime}, \theta^{\prime}, \alpha^{\prime}\right)=R R(\varphi, \theta, \alpha) R_{3}^{-1}$. 
Hereby it results that the Cartesian coordinates transform under rotations $R \in S O(3)$ as

$$
\begin{aligned}
& \vec{x} \rightarrow \vec{x}^{\prime}=R \vec{x}, \\
& y \rightarrow y^{\prime}=y+h(R, \vec{x}),
\end{aligned}
$$

where the function $h$ is given in Ref. 14. Thus, the vector $\vec{x}$ transforms according to an usual linear representation but the transformation of the fourth Cartesian coordinate is governed by a representation of $S O(3)$ induced by $\mathrm{SO}_{3}(2)$. The transformations of the group $\mathrm{SO}_{3}(2) \sim U(1)$ affect only the fourth coordinate translating it.

In this context, we observe that the 1-forms

$$
d \Omega(\varphi, \theta, \alpha)=R(\varphi, \theta, \alpha)^{-1} d R(\varphi, \theta, \alpha) \in \operatorname{so}(2)
$$

transform independently on $R$ as

$$
\left(R, R_{3}\right): d \Omega(\varphi, \theta, \alpha) \rightarrow d \Omega\left(\varphi^{\prime}, \theta^{\prime}, \alpha^{\prime}\right)=R_{3} d \Omega(\varphi, \theta, \alpha) R_{3}^{-1},
$$

finding that, beside the trivial quantity $d s_{1}{ }^{2}=d r^{2}$, there are two types of line elements invariant under $\mathbf{I}\left(M_{4}\right)$,

$$
\begin{aligned}
& d s_{2}{ }^{2}=-\left\langle d \Omega(\varphi, \theta, \alpha)^{2}\right\rangle_{33}=d \theta^{2}+\sin ^{2} \theta d \varphi^{2}, \\
& d s_{3}{ }^{2}=-\frac{1}{2} \operatorname{Tr}\left[d \Omega(\varphi, \theta, \alpha)^{2}\right]=d \theta^{2}+\sin ^{2} \theta d \varphi^{2}+(d \alpha+\cos \theta d \varphi)^{2} .
\end{aligned}
$$

Here it is worth pointing out that the above metrics are related to a family of metrics on the spheres $S^{3} \sim S O(3)$ parametrized with the angle variables $(\theta, \varphi, \alpha)$ with $0 \leq \theta<\pi, 0 \leq \varphi<2 \pi$ and $0 \leq \alpha<4 \pi$. The Hopf fibration $\left(S^{3}, d s_{3}\right) \rightarrow\left(S^{2}, d s_{2}\right)$ defines a vertical subbundle and its orthogonal complement with respect to the standard metric (6). The restrictions of this metric to the horizontal, respectively the vertical bundle, give the horizontal line element $d s_{h}{ }^{2}=d s_{2}{ }^{2}$ and the vertical one

$$
d s_{v}{ }^{2}=d s_{3}{ }^{2}-d s_{2}{ }^{2}=(d \alpha+\cos \theta d \varphi)^{2} .
$$

The conclusion is that the manifolds $\left(M_{4}, d s\right)$ with the isometry group $\mathbf{I}\left(M_{4}\right)$ have metrics defined by the line elements of the general form,

$$
d s^{2}=d s_{o}{ }^{2}+f_{v}(r) d s_{v}{ }^{2}, \quad d s_{o}{ }^{2}=f(r) d r^{2}+f_{h}(r) d s_{h}{ }^{2},
$$


where $f, f_{h}$ and $f_{v}$ are arbitrary functions only on $r$. From the physical point of view these manifolds represent backgrounds of Abelian Kaluza-Klein theories where the physical spaces (without gauge fields) are the base manifolds of the fibrations $\left(M_{4}, d s\right) \rightarrow\left(M_{3}, d s_{o}\right)$ induced by the Hopf one. The fiber results to be isomorphic with the little group $S_{3}(2) \sim U(1)$ which plays the role of the gauge group of these Abelian theories. The form of the vertical metric (7) in Cartesian coordinates, $d s_{v}{ }^{2}=\left(d y+A_{i} d x^{i}\right)^{2}$, emphasizes of the potentials $A_{i}$ of the Dirac magnetic monopole.

Hereby the lesson we have to learn is that some Kaluza-Klein theories could be obtained directly constructing the corresponding geometries with an appropriate symmetry. The main point is that the physical angular coordinates $(\theta, \varphi)$ and the Kaluza-Klein extra-coordinate $\alpha$ form a set of Euler parameters of the $S O(3)$ group. This allowed us to define the isometries in integral form and to find the general expression of the invariant line elements (8). It is important to observe that the Hopf fibration, involving only spheres, removes the string singularities of the potentials $A_{i}$ in all the geometries of this class of symmetry, independent on the choice of the invariant functions $f, f_{h}$ and $f_{v}$.

This conjecture can be generalized to non-Abelian Kaluza-Klein theories with $n$-dimensional physical spaces. We shall assume that in the whole manifolds of these theories there exist orbits $\Sigma \sim S O(n)$ whose coordinates, including the extra-dimensions, form sets of parameters of the groups $S O(n)$. In any physical space, we have to take a string with the corresponding little group $S O(n-1)$ that preserves its direction and defines the orbits $\Sigma_{o} \sim S O(n) / S O(n-1)$ of the physical space. Then we can exploit the fibrations $\Sigma \rightarrow \Sigma_{o}$ whose fibers $F \sim S O(n-1)$ are isomorphic with the little groups. In this framework the isometries can be introduced postulating similar automorphysms as in the previous particular case. Thus we may obtain $S O(n) \otimes S O(n-1)$ isometries and associated gauge transformations which should assure the central symmetry of the whole theory and convenient nontrivial fiberings. These geometries have to give rise to particular Yang-Mills potentials that may be written down separating the vertical projections of the metrics in the tangent spaces of the principal bundles $\Sigma$. We note that in this approach we must consider, in addition, the associated spin bundles whose structure groups are the universal covering groups $\operatorname{Spin}(n-1)$ of the structure groups $S O(n-1)$ of the principal bundles.

In what follows we should like to develop this theory for any dimensions and pseudo-orthogonal groups. 


\section{The general framework}

Let us start with the vector space $M_{n} \sim \mathbb{R}^{n}$ equipped with the associated (pseudo)-Euclidean metric $\eta$ of an arbitrary signature $\left(n_{+}, n_{-}\right)$with $n_{+}+$ $n_{-}=n$ that for $n_{-}=0$ becomes Euclidean. We denote by $x^{\mu}, \alpha, \ldots, \mu, \nu, \ldots=$ $1,2, \ldots, n$ the components of a vector $x=\left(x^{1}, x^{2}, \ldots, x^{n}\right)^{T}$ and consider the bilinear form ( . ) : $M_{n} \times M_{n} \rightarrow \mathbb{R}$ defined by $\eta$ that can be expressed in the matrix notation as $\left(x \cdot x^{\prime}\right)=x^{T} \eta x^{\prime}$. This form remains invariant under the transformations, $G: x \rightarrow G x, \forall x \in M_{n}$, of the fundamental representation of the (pseudo)-orthogonal group $\mathbf{G}=S O\left(n_{+}, n_{-}\right)$since the matrices $G \in \mathbf{G}$ obey $G^{T} \eta G=\eta$. We assume that $M_{n}$ is orientable, the improper transformations of the group $O\left(n_{+}, n_{-}\right)$changing the chirality of its Cartesian frames. When $(x \cdot x)>0$ we can use the unit vector $\hat{x}$ defined

such that $x=|x| \hat{x}$ where the invariant $|x|=\sqrt{(x \cdot x)}$ becomes the norm of $x$ in the Euclidean case.

\subsection{Groups and orbits}

In $\mathbf{G}$ one can introduce many types of parametrizations but in practice it is convenient to work with the standard covariant parametrization (of the first kind),

$$
G(\omega)=e^{X(\omega)}, \quad X(\omega)=\frac{1}{2} X_{(\alpha \beta)} \omega^{\alpha \beta}, \quad \forall G \in \mathbf{G}
$$

with real skew-symmetric parameters $\omega^{\mu \nu}=-\omega^{\nu \mu}$ and real basis-generators $X_{(\mu \nu)}=-X_{(\nu \mu)}$ whose properties are briefly presented in the Appendix A. The matrices $X(\omega)$ form the fundamental representation of the Lie algebra $\mathbf{g}=s o\left(n_{+}, n_{-}\right)$of the group $\mathbf{G}$ carried by the vector space $M_{n}$. When one uses other parametrizations one can express $\omega=\omega(z)$ in terms of the new parameters $(z)$ and work with the generators $X(z)=X[\omega(z)]$ without to change the basis-generators of $\mathbf{g}$. The matrices $X(z)$ have to generate the transformations matrices $G(z)=G[\omega(z)]$ according to Eq. (9). However, other types of parametrizations similar to the Euler one will be also used. In any parametrization $G(0)=1_{n}$ is the identity matrix of $\mathbf{G}$.

For our further developments the 1-forms $d \Omega(G)=G^{-1} d G$ defined for any $G \in \mathbf{G}$ are of a major importance. These are elements of the Lie algebra g that satisfy

$$
d \Omega\left(G^{\prime} G\right)=d \Omega(G)+G^{-1} d \Omega\left(G^{\prime}\right) G, \quad d \Omega\left(G^{-1}\right)=-G d \Omega(G) G^{-1}
$$


and have the supplemental property

$$
d \Omega(G)^{T}=-\eta d \Omega(G) \eta,
$$

resulting from the fact that $G$ are (pseudo)-orthogonal matrices.

It is know that, in general, any Lie group $\mathbf{G}=S O\left(n_{+}, n_{-}\right)$is isomorphic with a manifold $\Sigma$ of dimension $\frac{1}{2} n(n-1)$ which is an hyperboloid for an arbitrary signature $\left(n_{+}, n_{-}\right)$or a sphere in the Euclidean case when $\eta=1_{n}$ and $\mathbf{G}=S O(n)$ is an orthogonal group. This means that a given parametrization $(z)$ of $\mathbf{G}$ defines a system of coordinates in a local chart of $\Sigma \sim \mathbf{G}$. The general expression of the line element in this chart is given by the Killing metric as

$$
d s_{Z}^{2}=-\operatorname{Tr}\left\{Z d \Omega[G(z)]^{2}\right\}
$$

where $Z=Z^{\dagger}$ is a Hermitian non-singular matrix which plays the role of a metric operator. In other respects, the manifolds $\Sigma$ can be seen as being embedded in a larger space $M_{N} \sim \mathbb{R} \times \Sigma \sim \mathbb{R}^{N}$ of dimension $N=\frac{1}{2} n(n-1)+1$ where the set of shells $\Sigma$ of different radius may represent a foliation of a several domain of $M_{N}$ or even of the whole space $M_{N}$ when the metric $\eta$ is Euclidean and $\Sigma \sim S^{N-1}$ are spheres.

Similarly, we consider the orbits of the group $\mathbf{G}$ in the carrier space $M_{n}$. For the fixed vector $x_{o}$ with $\left(x_{o} \cdot x_{o}\right)>0$ there exists the associated orbit $\Sigma_{o}=\left\{x \mid x=G x_{o}, \forall G \in \mathbf{G}\right\} \subset M_{n}$ formed by all the vectors $x$ with the same norm $|x|=\left|x_{o}\right|$. The transformations $G_{o}$ that leave $x_{o}$ invariant constitute the little group $\mathbf{G}_{o}=\left\{G_{o} \mid G_{o} x_{o}=x_{o}\right\}$ of the orbit $\Sigma_{o}$ which will be supposed to be a compact subgroup of $\mathbf{G}$. All the orbits $\Sigma_{o}$ of different radius are isomorphic with the coset space $\mathbf{G} / \mathbf{G}_{o}$ and, therefore, $\operatorname{dim} \Sigma_{o}=n-1$. In general, these are hyperboloids but when the metric is Euclidean and $\mathbf{G}$ is compact then the orbit $\Sigma_{o} \sim S^{n-1}$ is a sphere of radius $\left|x_{o}\right|$ in $M_{n}$. In this case $\Sigma_{o}$ is covered by two local charts, one for the upper hemisphere $\Sigma_{o}^{+}$with the pole in $x_{o}$ and another for the lower hemisphere $\Sigma_{o}^{-}$whose pole is in $-x_{o}$. We say that the $n-1$ parameters defining the unit vector $\hat{x}$ form the Cartesian coordinates $(\hat{x})$ of the orbit $\Sigma_{o}$ understanding that for the spherical orbits we use the same coordinates in the domain $\Sigma_{o}^{+} \cap \Sigma_{o}^{-}$.

We have seen that the central pieces of the geometries we study here are the string along the direction of $\hat{x}_{o}$ and the corresponding little group $\mathbf{G}_{o}$. This suggests us to consider the well-known fibration $\mathbf{G} \rightarrow \mathbf{G} / \mathbf{G}_{o}$ that corresponds to the fibration $\Sigma \rightarrow \Sigma_{o}$ the base of which is the orbit $\Sigma_{o}$ of the space $M_{n}$. In this way the orbit $\Sigma$ becomes a principal fiber bundle with the 
fiber $F \sim \mathbf{G}_{o}$. This fibering splits the tangent space $\mathcal{T}(\Sigma) \sim \mathbf{g}$ of $\Sigma$ into a vertical subbundle and its horizontal complement. In terms of Lie algebras, the vertical projection is the Lie algebra $\mathbf{g}_{o}$ of $\mathbf{G}_{o}$ which is a subalgebra in $\mathbf{g}$ while the horizontal complement is the coset space $\mathbf{g} / \mathbf{g}_{o}$.

These may be easily manipulated if we introduce the elementary projection operator $P_{o}$ on the one-dimensional subspace of $x_{o}$, with the obvious properties $P_{o} x_{o}=x_{o}$ and $P_{o} G_{o}=G_{o} P_{o}=P_{o}$ for any $G_{o} \in \mathbf{G}_{o}$. Since $P_{o}$ is an elementary projection operator it gives the signature $\epsilon_{o}$ of the direction $\hat{x}_{o}$ as

$$
P_{o} \eta=\eta P_{o}=\epsilon_{o} P_{o} .
$$

The operator $P_{o}$ allows us to separate the elements of the Lie algebra $\mathbf{g}_{o}$. Thus any generator $X \in \mathrm{g}$ admits the orthogonal decomposition, $X=X_{v}+X_{h}$, in its vertical and horizontal parts expressed as

$$
\begin{aligned}
& X_{v}=\left(1_{n}-P_{o}\right) X\left(1_{n}-P_{o}\right)=X-P_{o} X-X P_{o} \in \mathbf{g}_{o}, \\
& X_{h}=P_{o} X\left(1_{n}-P_{o}\right)+\left(1_{n}-P_{o}\right) X P_{o}=P_{o} X+X P_{o} \in \mathbf{g} / \mathbf{g}_{o} .
\end{aligned}
$$

Here we used the property that $P_{o} X P_{o}=0$ for all $X \in \mathrm{g}$ since $P_{o}$ is an elementary projection operator and $X$ has no diagonal elements. Other useful calculation formulas are

$$
\operatorname{Tr}\left(X_{h}^{2}\right)=2 \operatorname{Tr}\left(P_{o} X^{2}\right), \quad \operatorname{Tr}\left(X_{v}^{2}\right)=\operatorname{Tr}\left(I_{o} X^{2}\right),
$$

where $I_{o}=1_{n}-2 P_{o}$ is the reflexion transformation of the group $O\left(n_{+}, n_{-}\right)$ which changes the sign of the string axis, $I_{o} x_{o}=-x_{o}$.

\subsection{Cartesian coordinates}

The orbit $\Sigma_{o}$ may be generated either using arbitrary transformations of $\mathbf{G}$ or explicitly exploiting a fixed isomorphism $B: \Sigma_{o} \rightarrow \mathbf{G} / \mathbf{G}_{o}$. This gives the "boosts" matrices $B(\hat{x})$, that transform $x_{o}$ into a desired vector $x=B(\hat{x}) x_{o}$. Since $|x|=\left|x_{o}\right|$ the matrix $B(\hat{x})$ depends only on the $n-1$ parameters of $\hat{x}$ which are just the coordinates of the Cartesian chart $(\hat{x})$ of the orbit $\Sigma_{o}$. We assume that the boost matrices have the form

$$
B(\hat{x})=e^{\lambda X(\hat{x})} \in \mathbf{G} / \mathbf{G}_{o},
$$

depending on the scalar function $\lambda=\lambda(\hat{x})$ and the horizontal generator defined by

$$
X(\hat{x})=\frac{X_{(\alpha \beta)} \hat{x}^{\alpha} \hat{x}_{o}^{\beta}}{\sqrt{\left|1-\left(\hat{x} \cdot \hat{x}_{o}\right)^{2}\right|}} \in \mathbf{g} / \mathbf{g}_{o},
$$


for all $\hat{x} \neq \hat{x}_{o}$ whereas $X\left(\hat{x}_{o}\right)=0$. Their properties given in the Appendix A allow us to find the closed matrix form

$$
B(\hat{x})= \begin{cases}1_{n}+X(\hat{x})^{2}(1-\cos \lambda)+X(\hat{x}) \sin \lambda & \text { if } \quad\left(\hat{x} \cdot \hat{x}_{o}\right)<1, \\ 1_{n}+X(\hat{x})^{2}(\cosh \lambda-1)+X(\hat{x}) \sinh \lambda & \text { if } \quad\left(\hat{x} \cdot \hat{x}_{o}\right)>1\end{cases}
$$

where in the first case we identify $\cos \lambda=\left(\hat{x} \cdot \hat{x}_{o}\right)$ while in the second one we must take $\cosh \lambda=\left(\hat{x} \cdot \hat{x}_{o}\right)$. In other words $B(\hat{x})$ can be either a rotation of angle $\lambda$ or a Lorentz-type boost. These boosts will help us to calculate the line elements of the charts with Cartesian coordinates $(\hat{x})$ of $\Sigma_{o}$ in terms of the 1-forms

$$
d \Omega[B(\hat{x})]=d \Omega[B(\hat{x})]_{h}+d \Omega[B(\hat{x})]_{v},
$$

whose projections are

$$
d \Omega[B(\hat{x})]_{h}= \begin{cases}d \lambda X(\hat{x})+\sin \lambda d X(\hat{x}) & \text { if } \quad\left(\hat{x} \cdot \hat{x}_{o}\right)<1 \\ d \lambda X(\hat{x})+\sinh \lambda d X(\hat{x}) \quad \text { if } \quad\left(\hat{x} \cdot \hat{x}_{o}\right)>1\end{cases}
$$

and

$$
d \Omega[B(\hat{x})]_{v}=\left\{\begin{array}{lll}
(1-\cos \lambda)[d X(\hat{x}), X(\hat{x})] & \text { if } \quad\left(\hat{x} \cdot \hat{x}_{o}\right)<1 \\
(\cosh \lambda-1)[d X(\hat{x}), X(\hat{x})] & \text { if } \quad\left(\hat{x} \cdot \hat{x}_{o}\right)>1
\end{array}\right.
$$

We note that these formulas were obtained taking into account that for any generator $X=X(\hat{x})$ we have $X d X X=(\hat{x} \cdot d \hat{x}) X=0$. Then using the general properties (10), (111) and (13) we find the invariant line element

$$
\begin{aligned}
d s_{h}{ }^{2} & =(d \hat{x} \cdot d \hat{x})=-\hat{x}_{o}^{T} \eta d \Omega[B(\hat{x})]^{2} \hat{x}_{o} \\
& =-\epsilon_{o} \operatorname{Tr}\left\{P_{o} d \Omega[B(\hat{x})]^{2}\right\}=-\frac{\epsilon_{o}}{2} \operatorname{Tr}\left\{d \Omega[B(\hat{x})]_{h}{ }^{2}\right\} .
\end{aligned}
$$

The Cartesian coordinates of the orbit $\Sigma$ will be defined bearing in mind that $\Sigma$ is isomorphic with $\mathbf{G}$. Therefore, we introduce the Cartesian coordinates $(\hat{x}, y)$ in a local chart of $\Sigma$ using the following parametrization of the group $\mathbf{G}$

$$
G(\hat{x}, y)=B(\hat{x}) G_{o}(y),
$$

where $(y)$ represents a set of $m=\frac{1}{2}(n-1)(n-2)$ arbitrary parameters of the group $\mathbf{G}_{o}$. In these coordinates the metric of $\Sigma$ is given by a line element of the general form (12) where we must take $(z) \equiv(\hat{x}, y)$. Then, we say that Eq. (23) defines the horizontal projection of this metric. 
We have introduced the parametrization (24) in accordance to the fibration $\Sigma \rightarrow \Sigma_{o}$ with the base $\Sigma_{o}$. Whenever the group $\mathbf{G}$ is not compact the orbit $\Sigma_{o}$ may have different disjoint shells each one being covered by its own chart and, therefore, the fibration is trivial. In the compact case the situation is more complicated since then the orbit $\Sigma_{o}$ is a sphere with at least two local charts where we can take different parameters of the fiber $F$. Therefore, we introduce the Cartesian coordinates $\left(\hat{x}, y^{+}\right)$for the upper hemisphere $\Sigma_{o}^{+}$ and $\left(\hat{x}, y^{-}\right)$for the lower one, $\Sigma_{o}^{-}$. On the overlapping domain $\Sigma_{o}^{+} \cap \Sigma_{o}^{-}$these coordinates must be related among themselves through the transition

$$
G_{o}\left(y^{-}\right)=T(\hat{x}) G_{o}\left(y^{+}\right), \quad T(\hat{x}) \in \mathbf{G}_{o} .
$$

The section $T: \Sigma_{o} \rightarrow \mathbf{G}_{o}$ is the transition function [2] of the fibration $\Sigma \rightarrow \Sigma_{o}$ which determines the topological properties of the principal bundle $\Sigma$, including its homotopy type.

\subsection{Spherical coordinates}

Other important systems of coordinates of $\Sigma$ involved in applications are the spherical coordinates. These have to be introduced observing that the above boosts can be written as

$$
B(\hat{x})=G_{o}(\theta) B_{s}(\lambda) G_{o}(\theta)^{-1},
$$

where $B_{s}(\lambda)$ is a one-parameter transformation matrix of the form (17) generated by the fixed horizontal generator $X_{s} \in \mathbf{g} / \mathbf{g}_{o}$ and arbitrary $\lambda$. This transforms $\hat{x}_{o}$ into the unit vector $\hat{x}(\lambda)=B_{s}(\lambda) \hat{x}_{o}$ while $G_{o}(\theta) \in \mathbf{G}_{o}$ performs the rotation $\hat{x}=G_{o}(\theta) \hat{x}(\lambda)$. There are $n-2$ parameters $(\theta)$ chosen to be just the generalized spherical coordinates of the sphere $S^{n-2}$ and only one parameter of $B_{s}(\lambda)$. These form the angular parameters of $\hat{x}=\hat{x}(\theta, \lambda)$ and, therefore, represent the spherical coordinates $(\theta, \lambda)$ of the orbit $\Sigma_{o}$. Let us observe that, by definition, the generators $X(\hat{x})$ do not depend explicitly on $\lambda$ and, consequently, we can write

$$
X[\hat{x}(\theta, \lambda)]=G_{o}(\theta) X_{s} G_{o}(\theta)^{-1} .
$$

In order to fill in the set of the angular coordinates of $\Sigma$, we introduce other $m$ angular variables $(\alpha)$ as parameters of $\mathbf{G}_{o}$ such that the corresponding parametrization of $\mathbf{G}$ should be given by

$$
G(\theta, \lambda, \alpha)=G_{o}(\theta) B_{s}(\lambda) G_{o}(\alpha) .
$$


This parametrization defines the spherical chart $(\theta, \lambda, \alpha)$ of $\Sigma$ related to the Cartesian one, $(\hat{x}, y)$, according to Eq. (26) and the obvious condition $G(\hat{x}, y)=G(\theta, \lambda, \alpha)$ which leads to the rule

$$
G_{o}(y)=G_{o}(\theta) G_{o}(\alpha)
$$

giving the coordinates $(y)$ in terms of the angular coordinates $(\theta)$ and $(\alpha)$. We specify that for the matrices $G_{o}(y)$ and $G_{o}(\alpha)$ we can use either the same type or even completely different types of parametrizations.

The invariant line element (23) in spherical coordinates reads,

$$
d s_{h}{ }^{2}=-\epsilon_{o} \operatorname{Tr}\left\{P_{o} d \Omega\left[G_{o}(\theta) B_{s}(\lambda)\right]^{2}\right\}
$$

This can be evaluated using Eqs. (10) and bearing in mind that $X_{s}$ is fixed so that $d \Omega\left[B_{s}(\lambda)\right]=d \lambda X_{s}$. Then, after a few manipulations we find

$$
d s_{h}{ }^{2}=\epsilon_{o}\left\{\begin{array}{lll}
d \lambda^{2}+\sin ^{2} \lambda d \theta^{2} & \text { for } & \left(\hat{x}_{s} \cdot \hat{x}_{o}\right)<1, \\
-\left(d \lambda^{2}+\sinh ^{2} \lambda d \theta^{2}\right) & \text { for } & \left(\hat{x}_{s} \cdot \hat{x}_{o}\right)>1
\end{array}\right.
$$

where $d \theta^{2}=-\frac{1}{2} \operatorname{Tr}\left[G_{o}(\theta)^{2}\right]$ is the standard line element in spherical coordinates of the sphere $S^{n-2}$.

In the case of the spherical orbits $\Sigma_{o}$ we can take different spherical coordinates for each hemisphere associating the coordinates $\left(\theta, \lambda, \alpha^{ \pm}\right)$to the Cartesian ones $\left(\hat{x}, y^{ \pm}\right)$. These are related within the transition

$$
G_{o}\left(\alpha^{-}\right)=T_{s}(\theta, \lambda) G_{o}\left(\alpha^{+}\right)
$$

which is equivalent to the transition (25) of the Cartesian charts if

$$
T_{s}(\theta, \lambda)=G_{o}(\theta)^{-1} T[\hat{x}(\theta, \lambda)] G_{o}(\theta)
$$

\section{Isometries and gauge transformations}

As mentioned, one of our major objectives is to find all the manifolds $\left(M_{N}, d s\right)$ of the Kaluza-Klein theories with the isometry group $\mathbf{I}\left(M_{N}, d s\right)=\mathbf{G} \otimes \mathbf{G}_{o}$ and to identify the Yang-Mills potentials arising in this context. To this end, we define the isometry transformations in closed form and we look for the gauge transformation of the Yang-Mills potentials produced by these isometries. 


\subsection{Isometries and invariant line elements}

In what concerns the isometry transformations, the main point of our approach is to postulate how transform the Cartesian coordinates $(\hat{x}, y)$ of $\Sigma$ under the group $\mathbf{G} \otimes \mathbf{G}_{o}$ which has to become the isometry group of $\Sigma$ and implicitly of $M_{N}$. Since the coordinates $(\hat{x}, y)$ are the parameters of the group $\mathbf{G}$ we can formulate the transformation laws exploiting some special automorphysms $\left(G, G_{o}\right): \mathbf{G} \rightarrow \mathbf{G}$ produced by the pairs $\left(G, G_{o}\right) \in \mathbf{G} \otimes \mathbf{G}_{o}$. First, we adopt the passive point of view keeping the manifold fixed and transforming among themselves the different charts covering the same domain. Furthermore, we assume that each pair $\left(G, G_{o}\right)$ transforms the Cartesian coordinates $(\hat{x}, y)$ into the new ones $\left(\hat{x}^{\prime}, y^{\prime}\right)$ such that the coordinates $(\hat{x})$ transform manifestly covariant as $\hat{x}^{\prime}=G \hat{x}$ and

$$
\left(G, G_{o}\right): G(\hat{x}, y) \rightarrow G\left(\hat{x}^{\prime}, y^{\prime}\right)=G G(\hat{x}, y) G_{o}^{-1} .
$$

Hereby and using Eq. (24) we obtain the definitive transformation rules

$$
\begin{aligned}
\hat{x} & \rightarrow \hat{x}^{\prime}=G \hat{x} \\
G_{o}(y) & \rightarrow G_{o}\left(y^{\prime}\right)=W(G, \hat{x}) G_{o}(y) G_{o}^{-1},
\end{aligned}
$$

where

$$
W(G, \hat{x})=B(G \hat{x})^{-1} G B(\hat{x})
$$

is a transformation matrix of $\mathbf{G}_{o}$ since $W(G, \hat{x}) \hat{x}_{o}=\hat{x}_{o}$. These matrices have a similar structure as the Wigner rotations of the representation theory of the Poincaré group in the momentum space [16], with the difference that in our case the matrices (37) depend on coordinates. Consequently, these have similar properties,

$$
W\left(G^{\prime} G, \hat{x}\right)=W\left(G^{\prime}, G \hat{x}\right) W(G, \hat{x}),
$$

and $W\left(G, \hat{x}_{o}\right)=1_{n}$ for any $G \in \mathbf{G}$ or $W\left(1_{n}, \hat{x}\right)=1_{n}$ for any $x$. Hereby we draw the conclusion that the coordinates $(y)$ transform according to a representation of the group $\mathbf{G}$ induced by the little group $\mathbf{G}_{o}$ [17. We note that the transformations (35) and (36) represent a combined transformation in the sense of Ref. [18]. These form a well-defined Lie group with some interesting properties which are presented in the Appendix B.

The next step is to find the metrics that remain invariant under these combined transformations. Since the 1 -forms $d \Omega[G(\hat{x}, y)] \in \mathbf{g}$ transform as

$$
\left(G, G_{o}\right): d \Omega[G(\hat{x}, y)] \rightarrow d \Omega\left[G\left(\hat{x}^{\prime}, y^{\prime}\right)\right]=G_{o} d \Omega[G(\hat{x}, y)] G_{o}^{-1}
$$


it is straightforward to show that the invariant metrics are the horizontal metric of $\Sigma_{o}$ given by the line element (23) and the metric of $\Sigma$ defined by Eq. (12) that in Cartesian coordinates reads

$$
d s_{Z}^{2}=-\operatorname{Tr}\left\{Z d \Omega[G(\hat{x}, y)]^{2}\right\}
$$

where $Z$ must be invariant under the transformations of $\mathbf{G}_{o}$ satisfying

$$
G_{o} Z G_{o}^{-1}=Z, \quad \forall G_{o} \in \mathbf{G}_{o}
$$

Therefore, $Z$ is a suitable linear combination of projection operators,

$$
Z=\alpha_{h} P_{o}+\frac{1}{2} \alpha_{v} I_{o}
$$

involving the arbitrary real constants $\alpha_{h}$ and $\alpha_{v}$. The invariant metrics (40) have specific horizontal and vertical parts that can be separated using the formula

$$
d \Omega[G(\hat{x}, y)]=G_{o}(y)^{-1}\left\{-d \Omega\left[G_{o}(y)^{-1}\right]+d \Omega[B(\hat{x})]\right\} G_{o}(y)
$$

resulting from Eqs. (10). We denote by

$$
d \omega(y)=-d \Omega\left[G_{o}(y)^{-1}\right]=\left[d G_{o}(y)\right] G_{o}(y)^{-1} \in \mathbf{g}_{o}
$$

the vertical 1-form depending on $y$ and split the 1 -form $d \Omega[B(\hat{x})]$ in its horizontal and vertical parts according to Eq. (20). Then we calculate the line element (40) with the metric operator (42) using Eqs. (16). The final result is

$$
d s_{Z}^{2}=\epsilon_{o} \alpha_{h} d s_{h}^{2}+\alpha_{v} d s_{v}^{2}
$$

where the line element of the vertical metric yields

$$
d s_{v}{ }^{2}=-\frac{1}{2} \operatorname{Tr}\left\{d \omega(y)+d \Omega[B(\hat{x})]_{v}\right\}^{2} .
$$

When $\alpha_{h}=\alpha_{v}=1$ the metric operator takes the standard form $Z=\frac{1}{2} 1_{n}$. However, the constants $\alpha_{h}$ and $\alpha_{v}$ can be replaced at any time by functions depending only on the invariant $|x|$, without to affect the symmetry of the line element.

These results lead to the conclusion that the manifolds $\left(M_{N}, d s\right)$ with the isometry group $\mathbf{I}\left(M_{N}, d s\right)=\mathbf{G} \otimes \mathbf{G}_{o}$ must have the Cartesian charts of coordinates $(x, y)$, with $x^{\mu}=|x| \hat{x}^{\mu}$, where the line elements

$$
d s^{2}=d s_{o}{ }^{2}+f_{v}(|x|) d s_{v}{ }^{2}, \quad d s_{o}{ }^{2}=f(|x|)(d|x|)^{2}+f_{h}(|x|) d s_{h}{ }^{2},
$$


depend on three arbitrary functions only on $|x|$, denoted by $f, f_{h}$ and $f_{v}$. In order to preserve the original signature of the metric $\eta$ it is recommended to take positive defined functions $f$ and $f_{h}$. In this metrization, the orbits $\Sigma$ and $\Sigma_{o}$ will get the metrics resulted from the restriction of the line elements (47) to fixed $|x|=\left|x_{o}\right|$.

The spherical charts of $\left(M_{N}, d s\right)$, denoted by $(|x|, \theta, \lambda, \alpha)$, have a radial coordinate $|x|$ and the angular coordinates $(\theta, \lambda, \alpha)$ of $\Sigma$ introduced above. The spherical coordinates are related to the Cartesian ones according to Eqs. (26) and (29). Consequently, the horizontal metric is given by Eq. (31) while the vertical line element (46) can be put in the form

$$
d s_{v}{ }^{2}=-\frac{1}{2} \operatorname{Tr}\left\{d \omega(\alpha)+\left[B_{s}(\lambda)^{-1} d \Omega\left[G_{o}(\theta)\right] B_{s}(\lambda)\right]_{v}\right\}^{2},
$$

where $d \omega(\alpha)=-d \Omega\left[G_{o}(\alpha)^{-1}\right]=\left[d G_{o}(\alpha)\right] G_{o}(\alpha)^{-1}$. In general, the separation

of the vertical part of the second term of $d s_{v}{ }^{2}$ is quite complicated depending on the concrete choice of $X_{s}$ and the angular variables $(\theta)$. However, this can be done in each particular case separately projecting the entire expression of this term on the subalgebra $\mathbf{g}_{o}$.

\subsection{Gauge transformations}

The manifolds $\left(M_{N}, d s\right)$ with the above isometry properties have to be considered as the principal fiber bundles of the $N$-dimensional Kaluza-Klein theories with central symmetry. The fibration $\left(M_{N}, d s\right) \rightarrow\left(M_{n}, d s_{o}\right)$ is induced by the fibration $\Sigma \rightarrow \Sigma_{o}$ so that the fiber bundles $\left(M_{N}, d s\right)$ and $\Sigma$ have the same fiber $F \sim \mathbf{G}_{o}$. For this reason the Kaluza-Klein extra-coordinates of the charts $(x, y)$ or $(\hat{x}, y)$ are the $m$ parameters $(y)$ of the little group $\mathbf{G}_{o}$. However, one can introduce other Cartesian charts, $\left(x, x_{K}\right)$ in $\left(M_{N}, d s\right)$ and $\left(\hat{x}, x_{K}\right)$ in $\Sigma$, with the new extra-coordinates, $x_{K}=\phi(y)$, related to the group parameters through the isomorphism $\phi: \mathbf{G}_{o} \rightarrow F$. In any case, when these fiberings are non-trivial we must provide appropriate transition functions relating the extra-coordinates of the different charts.

In the Kaluza-Klein theories the connection on the fiber $F$ is interpreted as a particular Yang-Mills potential which appears in the vertical part of the metric defined by the line element (47). We obtain thus physical models where the components of the Yang-Mills potential, $A_{\mu} \in \mathbf{g}_{o}$, are given by the term depending on $\hat{x}$ of the vertical metric (46). Consequently, we identify

$$
d A(x) \equiv A_{\mu}(x) d x^{\mu}=\frac{1}{2} X_{(\alpha \beta)} A_{\mu}^{(\alpha \beta)}(x) d x^{\mu}=d \Omega[B(\hat{x})]_{v},
$$


observing that these potentials are defined up to a real multiplicative constant playing the role of the coupling constant in the concrete physical models. More precisely, when one needs to work explicitly with the coupling constant $\kappa$ it is enough to rescale $f_{v} \rightarrow \kappa^{2} f_{v}$ and $y \rightarrow y / \kappa$ in Eq. (46).

A crucial problem is to find the non-Abelian gauge transformations of the Yang-Mills field represented by the above particular potentials. We believe that in these theories the gauge must have a natural geometrical origin with a simple physical significance. Let us observe that the boosts (17) giving rise to the Yang-Mills potentials (49) are determined up to an arbitrary transformation of $\mathbf{G}_{o}$ depending on coordinates. For this reason, we say that the section $H: \Sigma_{o} \rightarrow \mathbf{G}_{o}$ of the fiber bundle $\Sigma$ produces the gauge transformation of the boosts,

$$
B(\hat{x}) \rightarrow B^{\prime}(\hat{x})=B(\hat{x}) H(\hat{x})^{-1},
$$

generating the gauge transformations [2]

$$
d A(x) \rightarrow d A^{\prime}(x)=H(\hat{x}) d A(x) H(\hat{x})^{-1}+d \Omega\left[H(\hat{x})^{-1}\right]
$$

of the Yang-Mills potentials defined by Eq. (49).

The interpretation of the above gauge transformations is simple. We observe that Eq. (51) gives the form of the Yang-Mills potentials in the same domain but in a chart corresponding to the new parametrization $G\left(\hat{x}, y^{\prime}\right)=$ $\left[B(\hat{x}) H(\hat{x})^{-1}\right] H(\hat{x}) G_{o}(y) \in \mathbf{G}$. This is the Cartesian chart $\left(x, y^{\prime}\right)$ with the new extra-coordinates $y^{\prime}$ defined by $G_{o}\left(y^{\prime}\right)=H(\hat{x}) G_{o}(y)$. Thus $H$ plays the role of a transition function relating the extra-coordinates in the overlapping domain of the charts $(x, y)$ and $\left(x, y^{\prime}\right)$.

A remarkable property of the Yang-Mills potentials is that their transformations under isometries are combined with gauge transformations. More precisely, whenever one performs the isometry transformations (35) and (36) then the potentials (49) transform as

$$
\begin{aligned}
& \left(G, G_{o}\right): d A(x) \rightarrow d A(G x)=W(G, \hat{x}) d A(x) W(G, \hat{x})^{-1} \\
& +d \Omega\left[W(G, \hat{x})^{-1}\right],
\end{aligned}
$$

which means that $A(x)$ behaves like a vector field up to a gauge transformation produced by the new transformation matrices (37) we defined above. Therefore, we can say that the potentials (49) have the manifest central symmetry governed by the group G. We observe that our theory of isometries 
provides correct particular isometries $\left(1_{n}, G_{o}\right)$ of the fiber $F$ that do not involve gauge transformations.

After we have found the gauge transformations we are able to write down the fields strength

$$
F_{\mu \nu}=\partial_{\mu} A_{\nu}-\partial_{\nu} A_{\mu}+\left[A_{\mu}, A_{\nu}\right]
$$

that must satisfy the field equations

$$
\nabla^{\mu} F_{\mu \nu}+\left[A^{\mu}, F_{\mu \nu}\right]=j_{\nu}
$$

where $\nabla_{\mu}$ are the first $n$ covariant derivatives of $\left(M_{N}, d s\right)$ and $j_{\nu} \in \mathbf{g}_{o}$ is the current of the external sources. It is known that in the non-Abelian theories the gauge transformations (51) change the form of the fields strength,

$$
F_{\mu \nu}(x) \rightarrow F_{\mu \nu}^{\prime}(x)=H(\hat{x}) F_{\mu \nu}(x) H(\hat{x})^{-1} .
$$

For this reason the isometry transformations (152) which involve gauge transformations will transform the 2 -forms

$$
d F(x)=\frac{1}{2} F_{\mu \nu}(x) d x^{\mu} \wedge d x^{\nu}
$$

according to the rule

$$
\left(G, G_{o}\right): d F(x) \rightarrow d F(G x)=W(G, \hat{x}) d F(x) W(G, \hat{x})^{-1},
$$

leading to the conclusion that $F_{\mu \nu}$ transforms like a tensor field up to a gauge transformation. Hence we can say that all the models we have constructed here have a global central symmetry.

Finally, we specify that the presence of the spinor fields requires to consider, in addition, the spin bundle associated to the principal bundle $\left(M_{N}, d s\right)$. The structure group of the spin bundle is the gauge group of the entire theory which must be the universal covering group $\tilde{\mathbf{G}}_{o}$ of the little group $\mathbf{G}_{o}$. In other words, the gauge group is locally isomorphic with the little group, both these groups having the same algebra $\mathbf{g}_{o}$. Therefore, the gauge transformations (51) determine the transformations $\psi(x) \rightarrow U(\hat{x}) \psi(x)$ of the spinor fields whose unitary operators $U(\hat{x})=U[H(\hat{x})] \in \tilde{\mathbf{G}}_{o}$ have the same parametrization as $H(\hat{x})$ provided $U\left(1_{n}\right)=1_{s p}$ where $1_{s p}$ is the identity of $\tilde{\mathbf{G}}$. 


\subsection{Space-like and time-like models}

Our formalism becomes simpler if we take the string in a suitable fixed direction $x^{0}$ so that $\hat{x}_{o}=(1,0,0, \ldots, 0)$. Moreover, we take this direction of positive signature fixing $\eta_{00}=1$ and $\epsilon_{o}=1$. The other Cartesian coordinates of $\left(M_{n}, d s_{o}\right)$ will be denoted by $x^{i}, i, j, k, \ldots=1,2, \ldots, n-1$. In what concerns the spherical coordinates, a convenient choice is $X_{s}=X_{(n-10)}$ and

$$
G(\theta)=G_{s}\left(\theta_{1}\right) G_{s}\left(\theta_{2}\right) \ldots G_{s}\left(\theta_{n-2}\right),
$$

where $G_{s}\left(\theta_{j}\right)$ is a rotation of angle $\theta_{j}$ in the plane $\left(x^{j}, x^{j+1}\right)$, generated by $X_{(j j+1)}$. We must specify that the Euler angles used in the second section is not in accordance to this parametrization.

By definition the little group $\mathbf{G}_{o}$ acts only on the $n-1$ coordinates $x^{i}$ leaving the string direction $x^{0}$ invariant. In order to guarantee the unitarity of the Yang-Mills theory, the little group must be the orthogonal group $\mathbf{G}_{o}=$ $S O(n-1)$ since then the gauge group will be the compact group $\tilde{\mathbf{G}}_{o}=$ $\operatorname{Spin}(n-1)$. This means that the metric $\eta$ can be either $\eta=1_{n}$ or $\eta=$ $\operatorname{diag}\left(1,-1_{n-1}\right)$.

In the first case when $\eta=1_{n}$ all the Cartesian coordinates are spacelike including $x^{0}$ and we say that the model is space-like since its string is so. Obviously, all the space-like models are static such that physical models can be constructed only adding the time in a trivial manner. All the fields strength of these models are of magnetic type since $\left(M_{n}, d s_{o}\right)$ has no time-like coordinates.

However, when $\eta=\operatorname{diag}\left(1,-1_{n-1}\right)$ we have one time coordinate $x^{0}$ and $n-1$ space coordinates $x^{i}$ but the string is along the time direction $x^{0}$. The model with a string of this type will be called time-like model. In these models we have to meet fields of both electric and magnetic types. Interesting candidates for these models are the geometries having the same $S O(3,1)$ isometries as the Minkowski spacetime.

For the both types of models the matrices $X_{(i j)}$ are the vertical basisgenerators of the algebra $\mathbf{g}_{o}=s o(n-1)$ while the generators $X_{(i 0)}$ span the coset space $\mathbf{g} / \mathbf{g}_{o}$. In this context the definition (49) yields

$$
A_{k}=-\frac{X_{(k j)} x^{j}}{|x|\left(|x|+x^{0}\right)}, \quad A_{0}=0 .
$$

These potentials are defined up to gauge transformations that may dramatically change this very simple form. Obviously, the difference between the 
space-like and time-like models is encapsulated in the expression of $|x|$ as well as in the physical meaning of the coordinate $x^{0}$.

Each space-like or time-like model of a given dimension is determined by the concrete form of its invariant functions $f, f_{h}$ and $f_{v}$ and by the fibration that can be chosen independently on these functions. This means that there are many models with the same symmetry and Yang-Mills potentials (up to a gauge) but with different geometries. For this reason it is useful to divide the set of all of these models in classes of symmetry. We say that all the spacelike models with manifolds $\left(M_{N}, d s\right)$ of dimension $N=\frac{1}{2} n(n-1)+1$ and isometry group $\mathbf{I}\left(M_{N}, d s\right)=S O(n) \otimes S O(n-1)$ form the class of symmetry $\mathcal{S}(n)$. The time-like models of the same dimension but with the isometry group $\mathbf{I}\left(M_{N}, d s\right)=S O(1, n-1) \otimes S O(n-1)$ constitute the class of symmetry $\mathcal{S}(1, n-1)$.

The time-like models are completely new and seem to be rather special since these are no static and have extended singularities on the light-cone. For this reason we believe that these will rise new delicate problems that may be carefully analyzed elsewhere. In what follows we restrict ourselves to the space-like models of the classes $\mathcal{S}(n)$ which will be interpreted as generalized monopoles.

\section{Monopole models}

In the space-like models of the class $\mathcal{S}(n)$ the base manifolds $\left(M_{n}, d s_{o}\right)$ have only space-like Cartesian coordinates. Then the metric is $\eta=1_{n}$, the group $\mathbf{G}=S O(n)$ is orthogonal and the forms $(x \cdot x)=|x|^{2}=\left(x^{0}\right)^{2}+\eta_{i j} x^{i} x^{j}$ are positively defined. This justifies the usual notation $r=|x|$ for the radial coordinate of the spherical chart of $\left(M_{N}, d s\right)$ denoted from now by $(r, \theta, \lambda, \alpha)$. Here the line element is of the general form (47) where $d s_{h}{ }^{2}$ is given by the first of Eqs. (31) while the vertical part has to be calculated according to Eq. (48). In the Cartesian coordinates $(x, y)$ of the same manifold, the line element resulted from Eqs. (23), (46) and (49) is

$$
d s^{2}=f(r)(\hat{x} \cdot d x)^{2}+f_{h}(r)(d \hat{x} \cdot d \hat{x})-\frac{1}{2} f_{v}(r) \operatorname{Tr}\left[d \omega(y)+A_{\mu}(x) d x^{\mu}\right]^{2} .
$$

Let us observe that in our notation the Euclidean flat metric can be written as $d s_{E}^{2}=(d x \cdot d x)=(\hat{x} \cdot d x)^{2}+r^{2}(d \hat{x} \cdot d \hat{x})$. In general, the geometry of $\left(M_{N}, d s\right)$ is determined only by the choice of the invariant functions since the form of the potentials is given by Eq. (49). The most interesting case 
is when one can match these functions so that the metric of $\left(M_{N}, d s\right)$ be an exact solution of the vacuum Einstein equations.

\subsection{The monopole potentials}

The orbits $\Sigma_{o} \sim S^{n-1}$ of the models $\left(M_{N}, d s\right) \in \mathcal{S}(n)$ are spheres of fixed radius $r$. Here we have the pair of charts $\left(x, y^{ \pm}\right)$of the hemispheres $\Sigma_{o}^{ \pm}$ where the Yang-Mills potentials may take different forms $A^{ \pm}$. Obviously, on the domain $\Sigma_{o}^{+} \cap \Sigma_{o}^{-}$these must differ among themselves only within a gauge. Therefore, the potentials $A^{ \pm}$must be related to each other through a suitable gauge transformation (51) where if $A=A^{+}$then $A^{\prime}=A^{-}$. In order to construct an efficient mechanism of this type, it is indicated to consider the gauge transformation (52) associated to isometries rather than looking for arbitrary transition functions.

The group $O(n)$ has many proper or improper transformations able to change the sign of the axis $x_{o}$. All of them can be used for defining the gauge we need for our fiberings but here we consider only the proper transformations. Let us denote by $Q \in S O(n)$ a proper matrix that performs $Q x_{o}=-x_{o}$ and take the potentials of the upper hemispheres

$$
A_{k}^{+}(x)=-\frac{X_{(k i)} x^{i}}{r\left(r+x^{0}\right)}, \quad A_{0}=0
$$

Then the potentials of the lower hemispheres must be of the form

$$
A_{\mu}^{-}(x)=Q_{\cdot \mu}^{k \cdot} A_{k}^{+}(Q x)=-\frac{X_{(k i)} Q_{\cdot \mu}^{k \cdot} Q_{\cdot \nu}^{i \cdot} x^{\nu}}{r\left(r-x^{0}\right)}
$$

since these differ from $A_{k}^{+}$only through the gauge transformation (52) that now reads

$$
d A^{-}(x) \equiv d A^{+}(Q x)=T[Q](\hat{x}) d A^{+}(x) T[Q](\hat{x})^{-1}+d \Omega\left[T[Q](\hat{x})^{-1}\right],
$$

where $T[Q](\hat{x})=W(Q, \hat{x})$ can be calculated from Eqs. (37) and (88) obtaining

$$
T[Q](\hat{x})=Q\left[1_{n}+2 X(\hat{x})^{2}\right] .
$$

The mapping $T[Q]: \Sigma_{o} \rightarrow S O(n-1)$ is the transition function of the fibration that provides a suitable topological structure of the principal bundle $\left(M_{N}, d s\right)$, reducing the effects of the string singularity up to a point-like one. 
We note that the matrices $Q$ are defined up to left or/and right multiplications with arbitrary $S O(n-1)$ matrices that leave $x_{o}$ invariant. Each matrix $Q$ determines its specific fibration such that we have many possibilities of choice. However, these must be equivalent since the theory has a central symmetry.

In the spherical coordinates defined by Eqs. (28) and (158), the transition (32) is produced by an $S O(n-1)$ matrix which, according to Eqs. (27) and (33), depends only on the angular coordinates $(\theta)$ as

$$
T[Q]_{s}(\theta)=G_{o}(\theta)^{-1} Q G_{o}(\theta) B_{s}(\pi) .
$$

This formula becomes simpler if we take $Q=B_{s}(\pi)=1_{n}+2 X_{s}$ since then $B_{s}(\pi)$ commutes with the rotations $G_{s}\left(\theta_{j}\right)$ of Eq. (58) for all $j=1,2, \ldots, n-3$ while for $j=n-2$ we have $B_{s}(\pi) G_{s}\left(\theta_{n-2}\right) B_{s}(\pi)=G_{s}\left(-\theta_{n-2}\right)$. Therefore, we find that the transition functions of the spherical charts have the values

$$
T[Q]_{s}(\theta)=G_{s}\left(-2 \theta_{n-2}\right)
$$

for $0 \leq \theta_{n-2}<2 \pi$.

This result shows us that for all our space-like models the transition functions are simple rotations in the plane $\left\{x^{n-1}, x^{n-2}\right\}$ orthogonal to the string direction $x^{0}$. This means that the non-trivial part of the principal bundle $\Sigma$ reduces to the bundle over the two-dimensional sphere embedded in the Euclidean subspace $\left\{x^{0}, x^{n-1}, x^{n-2}\right\}$ where the transition function (66) provides $S O(2)$ rotations defined on the equatorial circle. Because of the central symmetry, this result can be reproduced for any other two-dimensional sphere $S_{i j}^{2}$ which surrounds the singularity at $x=0$, being embedded in the Euclidean subspace $\left\{x^{0}, x^{i}, x^{j}\right\}, i \neq j$. For this purpose it suffices to take an appropriate fibration whose transition function should be a rotation in the plane $\left\{x^{i}, x^{j}\right\}$.

In other respects, it is remarkable that the transition functions are defined on the angular domains $[0,4 \pi)$ that cover twice the equatorial circles. This assures the correct gauge transformations of the spinor fields whose gauge groups are simply connected. More precisely, the gauge transformations of the spinors corresponding to the rotations (66) are produced by the unitary transformations $U\left(-2 \theta_{n-2}\right) \in \operatorname{Spin}(n-1)$ which satisfy $U(-4 \pi)=1_{s p}$ when $\theta_{n-2}=2 \pi$. Otherwise, if this domain would be only $[0,-\pi)$ then the transformation $U(-2 \pi)=-1_{s p}$ might change the sign of spinors, the theory becoming thus pointless [19]. 
Hereby we conclude that the remaining point-like singularity behaves as a monopole since it is topologically stable [9] and, therefore, the topology of the principal bundle is of the homotopy type $\pi_{1}[S O(n-1)]=\mathbb{Z}_{2}$ if $n>3$. Thus the string singularities of the Yang-Mills potentials of all our non-Abelian models can be reduced up to monopoles in a similar manner as in the Abelian case of the Dirac magnetic monopole in the Taub-NUT background which is of the homotopy type $\pi_{1}[S O(2)]=\pi_{1}[U(1)]=\mathbb{Z}$ since $n=3$.

\subsection{The fields strength}

For understanding the behavior of the field represented by the above potentials, let us calculate the fields strength (153). In the case of the nonAbelian gauge theories the gauge transformations change the form of the fields strength without to affect the physical meaning of the theory. For this reason the potentials $A_{\mu}^{ \pm}$give rise to the different fields strength $F_{\mu \nu}^{ \pm}$related to each other within the gauge transformation (63) which yields

$$
d F^{-}(x) \equiv d F^{+}(Q x)=T[Q](\hat{x}) d F^{+}(x) T[Q](\hat{x})^{-1} .
$$

Consequently, it will suffice to calculate the components of the field $F_{\mu \nu} \equiv F_{\mu \nu}^{+}$ in terms of $A_{\mu} \equiv A_{\mu}^{+}$as

$$
\begin{aligned}
F_{0 i}(x) & =X_{(i k)} \frac{x^{k}}{r^{3}}, \\
F_{i j}(x) & =\frac{1}{r^{2}}\left[X_{(i j)}+A_{i}(x) x^{j}-A_{j}(x) x^{i}\right] .
\end{aligned}
$$

One can verify that these fields strength are exact solutions of the sourceless Yang-Mills equations (54). Starting with the obvious identities $x^{\mu} A_{\mu}=0$ and $x^{\mu} F_{\mu \nu}=0$ one finds $j_{0}=0$ which is compatible with the global $S O(n)$ symmetry only when $j_{\nu}=0$. Thus the requirements (I)-(III) are fulfilled remaining to study only the invariants.

The main differential forms related to the fields strength are the 2-form (56) and the dual $(n-2)$-form

$$
d F^{*}(x)=\frac{1}{2(n-2) !} F_{\alpha \beta}(x) \varepsilon_{. \cdot \sigma_{1} \ldots \sigma_{n-2}}^{\alpha \beta} d x^{\sigma_{1}} \wedge d x^{\sigma_{2}} \wedge \ldots d x^{\sigma_{n-2}},
$$

where $\varepsilon_{\alpha_{1} \alpha_{2} \ldots \alpha_{n}}$ is the total skew-symmetric $S O(n)$ tensor. With the help of $d F$ and $d F^{*}$ we can define the following candidates of invariants

$$
\Phi\left(S^{2}\right)=\int_{S^{2}} d F, \quad \Phi^{*}\left(S^{n-2}\right)=\int_{S^{n-2}} d F^{*} .
$$


These integrals are elements of the $s o(n-1)$ algebra representing fluxes through two-dimensional and, respectively, $(n-2)$-dimensional spheres of arbitrary radius $r_{0}$. We observe that the value of the first integral does not depend on $r_{0}$ so that the integration can be done over the unit sphere.

The orbit $\Sigma_{o}=S^{n-1}$ of unit radius embeds a number of $\frac{1}{2}(n-1)(n-2)=$ $m$ different unit spheres $S_{i j}^{2} \subset \Sigma_{o}$ surrounding the monopole. Therefore, we shall obtain $m$ different corresponding values of $\Phi$, each one depending on the $m$ basis-generators $X_{(i j)}$ of the $s o(n-1)$ algebra. In this manner we generate a real $m \times m$ matrix of flux components that can be put in diagonal form. Indeed, if we consider the complete system of $m$ spheres $S_{i j}^{2}$, $i, j=1,2, \ldots, n-1(i \neq j)$, then a simple calculation leads to the interesting result

$$
\Phi\left(S_{i j}^{2}\right)=4 \pi X_{(i j)}
$$

that holds for any space-like model. Since the generalized monopoles are topologically stable these fluxes represent the principal invariants of our space-like models.

In what concerns the second integral of (171) we observe that the number of spheres $S^{n-2} \subset S^{n-1}$ is $n-1$. In general, if $n \neq 4$ this number differs from $m$ which means that the number of integrals $\Phi^{*} \in s o(n-1)$ differs from that of the basis-generators of the $s o(n-1)$ algebra. This fact is not in agreement with the $S O(n-1)$ gauge symmetry, indicating that the fluxes $\Phi^{*}\left(S^{n-2}\right)$ may vanish. In the particular case of $n=4$ the concrete calculation leads to the same result as we shall see in the Example 2 of the next section.

For constructing second order invariants we can not use the 4-form $d F \wedge$ $d F$ since this is traceless. For this reason, we must consider the integrals

$$
\Theta(V)=-\frac{1}{2} \operatorname{Tr} \int_{V} d F(x) \wedge d F^{*}(x)
$$

over arbitrary volumes $V \subset M_{n}$. The singularity at $x=0$ of the fields strength makes these integrals divergent for the models with $n<5$ but if $n \geq 5$ and $M_{n}$ is a compact manifold then the quantity $\Theta\left(M_{n}\right)$ is a finite real number that may be interpreted as a second order invariant since, in general, this does not vanish.

The general conclusion is that the potentials (61) and (62) give rise to a field strength with $S O(n)$ central symmetry which is a particular solutions of the sourceless Yang-Mills equations in the $n$-dimensional physical space of a Kaluza-Klein theory with the gauge group $\operatorname{Spin}(n-1)$ and $S O(n) \otimes$ 
$S O(n-1)$ isometries. It is remarkable that this solution is topologically stable allowing as principal invariants the fluxes of the fields strength through the two-spheres surrounding the singularity at $x=0$. Since the values of these fluxes are $4 \pi$, in units of coupling constant, we can say that the space-like models presented here have the properties (I)-(IV) which, in our opinion, define a plausible version of generalized monopoles.

\subsection{Examples}

Our method of finding monopole solutions gives rise to a large collection of models divided in classes of symmetry $\mathcal{S}(n)$. Each of these classes is formed by all the models which have the same symmetry and implicitly the same gauge group. We believe that some of them could be attractive as can be seen from the table below which lists the first five classes of symmetry pointing out the dimensions, gauge groups [20] and fibrations.

\begin{tabular}{|c|c|c|c|}
\hline $\begin{array}{c}\text { symmetry } \\
\text { class } \mathcal{S}(n)\end{array}$ & $\begin{array}{c}\text { dimension } \\
\left(M_{N}, d s\right)\end{array}$ & $\begin{array}{c}\text { gauge group } \\
\mathrm{Spin}(n-1)\end{array}$ & $\begin{array}{c}\text { fibration } \\
\Sigma \rightarrow \Sigma_{o}\end{array}$ \\
\hline$n=3$ & $N=4$ & $U(1)$ & $S^{3} \rightarrow S^{2}$ \\
\hline$n=4$ & $N=7$ & $S U(2)$ & $S^{6} \rightarrow S^{3}$ \\
\hline$n=5$ & $N=11$ & $S U(2) \otimes S U(2)$ & $S^{10} \rightarrow S^{4}$ \\
\hline$n=6$ & $N=16$ & $S p(2)$ & $S^{15} \rightarrow S^{5}$ \\
\hline$n=7$ & $N=22$ & $S U(4)$ & $S^{21} \rightarrow S^{6}$ \\
\hline
\end{tabular}

In general, the models of a given class of symmetry have the same monopole fields but different geometries of the principal bundle $\left(M_{N}, d s\right)$ determined by the invariant functions $f, f_{h}$ and $f_{v}$. This offers one the opportunity to find new models that could be of some physical interest. Of course, first we look for the monopole models whose manifolds should be new solutions of the Einstein equations without matter terms. In what follows we restrict ourselves to discuss a well-known geometry and to present examples of new $S U(2)$ models with Einstein metrics.

\section{Example 1: The Euclidean Taub-NUT space}

A simple but famous example is the $U(1)$ Dirac magnetic monopole in the Euclidean Taub-NUT space. This is a special member of the class of symmetry $\mathcal{S}(3)$ we have presented in the second section as argument for our attempt. 
This has the virtue to be Ricci flat, its metric being an exact solution of the Einstein equations in vacuum. In the Cartesian charts $\left(\vec{x}, y^{ \pm}\right)$the line elements yield

$$
d s_{ \pm}^{2}=\frac{1+r}{r} d \vec{x} \cdot d \vec{x}+\frac{r}{1+r}\left(d y^{ \pm}+A_{i}^{ \pm} d x^{i}\right)^{2},
$$

where $A^{ \pm}$are the potentials of the Dirac magnetic monopole. Since the string is along the third axis we have $x^{0}=x^{3}$ and only one vertical generator, $X_{(12)}$. The potentials are defined by Eqs. (61) and (62) where we chose $Q=B_{s}(\pi)=R_{2}(\pi)=\operatorname{diag}(-1,1,-1)$ finding the components

$$
A_{1}^{ \pm}=\mp \frac{x^{2}}{r\left(r \pm x^{3}\right)}, \quad A_{2}^{ \pm}= \pm \frac{x^{1}}{r\left(r \pm x^{3}\right)}, \quad A_{3}^{ \pm}=0 .
$$

These give rise to the magnetic field with central symmetry

$$
\vec{B}=\operatorname{rot} \vec{A}^{ \pm}=\frac{\vec{x}}{r^{3}}
$$

In spherical coordinates, the set $(\theta)$ reduces to the angle $\varphi$, the angle $\theta$ plays the role of $\lambda$ and the only spherical extra-coordinate is $\alpha$. Therefore, we replace $G_{o}(\theta)$ with $R_{3}(\varphi)$ and $B_{s}(\lambda)$ with $R_{2}(\theta)$ taking $G_{o}(\alpha)=R_{3}(\alpha)$. Calculating the line elements according to our general method we obtain the well-known result

$$
d s_{ \pm}^{2}=\frac{1+r}{r}\left(d r^{2}+r^{2} d \theta^{2}+r^{2} \sin ^{2} \theta d \varphi^{2}\right)+\frac{r}{1+r}\left(d \alpha^{ \pm}+\cos \theta d \varphi\right)^{2},
$$

that may be derived directly from Eq. (174). Here the spherical coordinates $\alpha^{ \pm}$are related to each other according to Eq. (32) whose transition function (66) is now $T_{s}(\varphi)=R_{3}(-2 \varphi)$ so that $\alpha^{-}=\alpha^{+}-2 \varphi$, recovering thus a version of the Hopf fibration $S^{3} \rightarrow S^{2}$.

\section{Example 2: $S U(2)$ models}

Let us consider the case of $n=4$ and $N=7$ when the Kaluza-Klein manifolds $\left(M_{7}, d s\right) \in \mathcal{S}(4)$ are principal fiber bundles whose base manifolds, $\left(M_{4}, d s_{o}\right)$, have the associated flat metric $\eta=1_{4}$. In these models the little group is $S O(3)$ and the gauge group is $S U(2)$.

In general, a chart $(u)$ of $\left(M_{7}, d s\right)$ has the coordinates $u^{A}, A, B, \ldots=$ $0,1, \ldots, 6$. The Cartesian coordinates $(x, y)$ are formed by the physical ones, 
$(x)=\left(x^{0}, \vec{x}\right)$, and the arbitrary $S U(2)$ parameters, $(y)$. The coordinates $\vec{x}$ span the subspace $M_{3} \subset M_{4}$ orthogonal to the direction $x^{0}$. Since $M_{3}$ has the $S O(3)$ symmetry we can use here the vector notation taking into account that now $i, j, \ldots=1,2,3$. The corresponding spherical coordinates $(r, \theta, \varphi, \lambda)$ of the space $\left(M_{4}, d s_{o}\right)$ are defined by the transformation $x=G_{o}(\theta) B_{s}(\lambda) x_{o}$ with $G_{o}(\theta) \equiv R(\varphi, \theta, 0)$ while $B_{s}(\lambda)$ is a rotation of angle $\lambda$ in the plane $\left\{x^{0}, x^{3}\right\}$. Consequently, we have

$$
\begin{aligned}
& x^{0}=r \cos \lambda, \\
& x^{1}=r \sin \lambda \sin \theta \cos \varphi, \\
& x^{2}=r \sin \lambda \sin \theta \sin \varphi, \\
& x^{3}=r \sin \lambda \cos \theta .
\end{aligned}
$$

In addition, we introduce three spherical Kaluza-Klein extra-coordinates $(\alpha)$ denoted from now by $\alpha^{1}=\alpha, \alpha^{2}=\beta$ and $\alpha^{3}=\gamma$ which represent the Euler parameters of the rotations $G_{o}(\alpha) \equiv R(\alpha, \beta, \gamma) \in S O(3)$.

Following the general method, we start with the spherical charts of $\left(M_{7}, d s\right)$ having the coordinates $\left(u_{ \pm}\right) \equiv\left(r, \theta, \phi, \lambda, \alpha^{ \pm}, \beta^{ \pm}, \gamma^{ \pm}\right)$and the line elements

$$
\begin{aligned}
d s_{ \pm}^{2}= & g_{A B}\left(u_{ \pm}\right) d u_{ \pm}^{A} d u_{ \pm}^{B} \\
= & f(r) d r^{2}+f_{h}(r)\left[d \lambda^{2}+\sin ^{2} \lambda\left(d \theta^{2}+\sin ^{2} \theta d \varphi^{2}\right)\right] \\
& +f_{v}(r)\left\{\left(d \alpha^{ \pm}+\cos \beta^{ \pm} d \gamma^{ \pm}+\cos \theta d \varphi\right)^{2}\right. \\
& +\left(\sin \alpha^{ \pm} \sin \beta^{ \pm} d \gamma^{ \pm}+\cos \alpha^{ \pm} d \beta^{ \pm}+\cos \lambda d \theta\right)^{2} \\
& \left.+\left(\cos \alpha^{ \pm} \sin \beta^{ \pm} d \gamma^{ \pm}-\sin \alpha^{ \pm} d \beta^{ \pm}-\cos \lambda \sin \theta d \varphi\right)^{2}\right\} .
\end{aligned}
$$

Furthermore, we consider the non-trivial fibration defined by the transition function (66) that in this case yields $T[Q]_{s}(\theta)=R_{2}(-2 \theta)$ since $Q=B_{s}(\pi)=$ $\operatorname{diag}(-1,1,1,-1)$. The resulted fiber bundle $\left(M_{7}, d s\right)$ is non-trivial only on the sphere $S_{13}^{2}$. The transition transformation

$$
R\left(\alpha^{-}, \beta^{-}, \gamma^{-}\right)=R_{2}(-2 \theta) R\left(\alpha^{+}, \beta^{+}, \gamma^{+}\right)
$$

can be solved in terms of the Euler variables but the result is quite complicated.

The geometry of the principal bundles $\left(M_{7}, d s\right)$ is determined by the functions $f, f_{h}$ and $f_{v}$. Looking for models with metrics satisfying the vacuum Einstein equations, we find two solutions of the positive curvature 
$R_{A B}=6 k^{2} g_{A B}$

sol. $1: f(r)=3, \quad f_{h}(r)=\frac{1}{k^{2}} \sin ^{2} k r, \quad f_{v}(r)=\frac{1}{3 k^{2}} \sin ^{2} k r$,

sol. 2: $f(r)=5, \quad f_{h}(r)=\frac{1}{k^{2}} \sin ^{2} k r, \quad f_{v}(r)=\frac{1}{k^{2}} \sin ^{2} k r$,

and two solutions of the negative curvature $R_{A B}=-6 k^{2} g_{A B}$,

sol. $1: f(r)=3 \sec ^{2} k r, \quad f_{h}(r)=\frac{1}{k^{2}} \tan ^{2} k r, \quad f_{v}(r)=\frac{1}{3 k^{2}} \tan ^{2} k r$,

sol. 2 : $f(r)=5 \sec ^{2} k r, \quad f_{h}(r)=\frac{1}{k^{2}} \tan ^{2} k r, \quad f_{v}(r)=\frac{1}{k^{2}} \tan ^{2} k r ，$

where $k$ is a real constant. In both the above cases the limit $k \rightarrow 0$ leads to the Ricci flat metrics with $R_{A B}=0$,

$$
\begin{aligned}
& \text { sol. 1: } f(r)=3, \quad f_{h}(r)=r^{2}, \quad f_{v}(r)=\frac{1}{3} r^{2} \text {, } \\
& \text { sol. 2: } f(r)=5, \quad f_{h}(r)=r^{2}, \quad f_{v}(r)=r^{2} \text {. }
\end{aligned}
$$

All these results have been obtained using Maple.

In the Cartesian charts $\left(x, y^{ \pm}\right)$these solutions give the line elements of the form (60) with the Yang-Mills potentials (61) and, respectively, (62). The above fibering joints the descriptions of the gauge field in both these charts such that the physical effects depend only on the fields strength (68) and (69). These can be written in the vector notation if we use the $S O(3)$ generators $J_{i}=\frac{1}{2} \varepsilon_{i j k} X_{(j k)}$ and we put

$$
E_{i}=F_{0 i}, \quad B_{i}=\frac{1}{2} \varepsilon_{i j k} F_{j k} .
$$

Then we obtain

$$
\vec{E}=\frac{\vec{x} \times \vec{J}}{r^{3}}, \quad \vec{B}=\frac{x^{0} \vec{J}}{r^{3}}+\frac{\vec{x}(\vec{x} \cdot \vec{J})}{r^{3}\left(r+x^{0}\right)},
$$

where $r^{2}=\vec{x}^{2}+\left(x^{0}\right)^{2}$.

In this 4-dimensional model there exists the dual field strength $F_{\mu \nu}^{*}=$ $\frac{1}{2} \varepsilon_{\mu \nu \alpha \beta} F^{\alpha \beta}$ giving the 2 -form $d F^{*}$ defined by Eq. (70). The 2 -forms $d F$ and $d F^{*}$ help us to calculate the integrals (71) recovering the result (72) and $\Phi^{*}=0$ as was expected. In this model the integral (173) is divergent whereas 
other second order invariants can not be constructed since $\operatorname{Tr}(d F \wedge d F)=$ $\operatorname{Tr}\left(d F^{*} \wedge d F^{*}\right)=0$.

Even though it is premature to draw definitive conclusions, we observe that the values of these invariants as well as the fields (82) indicate that our $S U(2)$ models are new. It seems that these are closer to the BPS monopoles [8] rather than other models of $S U(2)$ monopoles [5, 6, 7].

\section{Concluding remarks}

Here we have shown that there are non-Abelian Kaluza-Klein geometries in $N$-dimensional principal fiber bundles with $S O(n) \otimes S O(n-1)$ isometries, having $n$-dimensional bases with manifest $S O(n)$ central symmetries and fibers $F \sim S O(n-1)$ representing the little groups the string directions. The Yang-Mills potentials (59) appear as being produced by strings but the string singularities can be reduced up to a point-like ones since the non-trivial principal bundles have suitable topological properties. Thus we obtain theories where the surviving singularities are topologically stable and, therefore, can be interpreted as generalized monopoles as long as the conditions (I)-(IV) are fulfilled. As a matter of fact, our approach is an inverse method which recovers the Yang-Mills fields from a given Kaluza-Klein geometry, avoiding to solve directly the Yang-Mills equations.

A remarkable result concerns the time-like models that are new to our knowledge. The Yang-Mills fields of these models are produced by time-like strings in physical backgrounds having one time-like coordinate and arbitrary space-like ones. The models of this type are no static and their Yang-Mills potentials are singular on the future light cone when the string is in the past. In this situation it is clear that the effects of this extended singularity can not be removed neither within topology nor using other methods. Thus we found the new classes of symmetry $\mathcal{S}(1, n-1)$ whose models seem to be rather special, with new and unusual properties. We hope that further investigations should clarify if these models could have a physical meaning.

From the technical point of view the central points of our approach are the parametrization (24) written with the help of the boosts (17) and the integral form of the isometry transformations (34) we postulated here. These lead to the induced representations (36) giving rise to the associated gauge transformations we needed for constructing models with central symmetry and the specific transition functions defining our fiberings. As observed in the 
section 4, these induced representations arise from an orbital analysis similar to the well-known one of the representation theory of the Poincaré group in the momentum space. The difference is that our induced representations are connected to the Kaluza-Klein geometry since their transformations depend on coordinates producing gauge transformations. This indicates that the study of these representations could emphasize new interesting mathematical properties.

Another mathematical challenge is how could be extended the above presented method of induced representations to theories with other types of singularities the reduction of which should require bundles of higher homotopy types, $\pi_{k}$ with $k>1$ [12]. A solution could be to replace the strings of our monopole models by branes.

\section{Acknowledgments}

I should like to thank Mihai Visinescu for helpful discussions and suggesting me to look for new monopole models with Einstein metrics. This work is partially supported by MEC-AEROSPATIAL Program, Romania.

\section{A The generators $X(\hat{x})$}

The real basis-generators $X_{(\mu \nu)}$ we use here have the matrix elements

$$
\left\langle X_{(\alpha \beta)}\right\rangle_{\cdot \nu}^{\mu \cdot}=\delta_{\alpha}^{\mu} \eta_{\beta \nu}-\delta_{\beta}^{\mu} \eta_{\alpha \nu}
$$

and the trace properties

$$
\operatorname{Tr}\left(X_{(\mu \nu)}\right)=0, \quad \operatorname{Tr}\left(X_{(\mu \nu)} X_{(\alpha \beta)}\right)=2\left(\eta_{\mu \beta} \eta_{\nu \alpha}-\eta_{\nu \beta} \eta_{\mu \alpha}\right)
$$

allowing one to define the Killing forms. They satisfy the commutation relations

$$
\left[X_{(\mu \nu)}, X_{(\sigma \tau)}\right]=\eta_{\mu \tau} X_{\nu \sigma}-\eta_{\mu \sigma} X_{\nu \tau}+\eta_{\nu \sigma} X_{\mu \tau}-\eta_{\nu \tau} X_{\mu \sigma},
$$

and transform as

$$
G X_{(\mu \nu)} G^{-1}=G_{\mu}^{\cdot \alpha} \cdot G_{\nu}^{\cdot \beta} X_{(\alpha \beta)} \cdot
$$

The boost generators defined by Eq. (18) have the obvious properties

$$
X(\hat{x})^{T}=s X(\hat{x}), \quad X(\hat{x})^{3}=s X(\hat{x}), \quad \operatorname{Tr}\left[X(\hat{x})^{2}\right]=2 s,
$$


where $s=\operatorname{sign}\left[\left(\hat{x} \cdot \hat{x}_{o}\right)^{2}-1\right]$. In the unitary case when $\left(\hat{x} \cdot \hat{x}_{o}\right)=\cos \lambda$, the transformations $Q \in S O(n)$ that satisfy $Q x_{o}=-x_{o}$ change $\lambda \rightarrow \pi-\lambda$ and give $X(Q \hat{x})=-Q X(\hat{x}) Q^{-1}$ such that

$$
B(Q \hat{x})=Q\left[1_{n}+X(\hat{x})^{2}(1+\cos \lambda)-X(\hat{x}) \sin \lambda\right] Q^{-1} .
$$

Hereby we find the important result (64).

\section{B Induced representations}

Let us start with the principal bundle $\Sigma$ with the fiber $F \sim \mathbf{G}_{o}$ and the base manifold $\Sigma_{o}$ where we choose the Cartesian chart $(\hat{x})$. We denote by $\operatorname{Sec}(\Sigma)$ the set of the (local) sections $V: \Sigma_{o} \rightarrow \mathbf{G}_{o}$ that form a group with respect to the multiplication $\times$ defined as $\left(V^{\prime} \times V\right)(\hat{x})=V^{\prime}(\hat{x}) V(\hat{x})$ for all $V, V^{\prime} \in$ $\operatorname{Sec}(\Sigma)$. The identity section obeys $\operatorname{Id}(\hat{x})=1_{n}$ and the inverse section of $V$ is $V^{-1}$ so that $V^{-1}(\hat{x})=[V(\hat{x})]^{-1}$. For any automorphism $\phi: \Sigma_{o} \rightarrow \Sigma_{o}$ we can define the sections $V \circ \phi$ with the obvious properties $\operatorname{Id} \circ \phi=\mathrm{Id}, V \circ \mathrm{id}=V$, when $\phi=$ id is the mapping identity, and $\left(V^{\prime} \times V\right) \circ \phi=\left(V^{\prime} \circ \phi\right) \times(V \circ \phi)$.

We denote by $\operatorname{Aut}\left(\Sigma_{o}\right)$ the group of automorphisms of $\Sigma_{o}$ and we say that the pair $(V, \phi)$, with $V \in \operatorname{Sec}(\Sigma)$ and $\phi \in \operatorname{Aut}\left(\Sigma_{o}\right)$, represents a combined transformation. These pairs constitute a group $\mathcal{G}$ with respect to the multiplication $*$ defined as follows:

$$
\left(V^{\prime}, \phi^{\prime}\right) *(V, \phi)=\left(\left(V^{\prime} \circ \phi\right) \times V, \phi^{\prime} \circ \phi\right) .
$$

The identity of this group is (Id,id) while the inverse of a pair $(V, \phi)$ reads

$$
(V, \phi)^{-1}=\left(V^{-1} \circ \phi^{-1}, \phi^{-1}\right) .
$$

One can verify that $\mathcal{G}=\operatorname{Sec}(\Sigma) \mathbf{s} \operatorname{Aut}\left(\Sigma_{o}\right)$ is a semidirect product where $\operatorname{Sec}(\Sigma)$ is the invariant subgroup [18].

The isometries of $\Sigma_{o}$ define the set of mappings $\phi_{G}$ as $\hat{x} \rightarrow \hat{x}^{\prime}=G \hat{x} \equiv$ $\phi_{G}(\hat{x})$ for all $G \in \mathbf{G}$. These form a group since $\phi_{G}^{\prime} \circ \phi_{G}=\phi_{G^{\prime} G},\left(\phi_{G}\right)^{-1}=\phi_{G^{-1}}$ and $\phi_{1_{n}}=\mathrm{id}$. In addition, we define the mapping $W: \mathbf{G} \rightarrow \operatorname{Sec}(\Sigma)$ whose values $W[G]$ are arbitrary sections. However, we can impose supplemental conditions such that the set of pairs $\left(W[G], \phi_{G}\right)$ should form a subgroup in $\mathcal{G}$. Indeed, if we assume that

$$
\left(W\left[G^{\prime}\right] \circ \phi_{G}\right) \times W[G]=W\left[G^{\prime} G\right], \quad W\left[1_{n}\right]=\mathrm{Id}
$$


then we find that the group multiplication,

$$
\left(W\left[G^{\prime}\right], \phi_{G^{\prime}}\right) *\left(W[G], \phi_{G}\right)=\left(W\left[G^{\prime} G\right], \phi_{G^{\prime} G}\right),
$$

defines the group $\mathcal{G}[\mathbf{G}] \subset \mathcal{G}$ of these pairs which is in fact a representation of the group $\mathbf{G}$ induced by $\mathbf{G}_{o}$ since $W[G](\hat{x})$ are elements of this group.

In the case of the matrices (37) we identify $W[G](\hat{x})=W(G, \hat{x})$ and from Eq. (38) it results that the condition (91) is fulfilled. The conclusion is that

the transformations (35) and (36) define an induced representation in the sense outlined above.

\section{References}

[1] M. F. Atyah, The Geometry of Yang-Mills Fields Fermi lectures (Scuola Normale, Pisa 1979).

[2] T. Eguchi, P. B. Gilkey and A. J. Hanson, Phys. Rep. 66 (1980) 214.

[3] P. A. M. Dirac, Proc. Roy. Soc. A 133 (1931) 60.

[4] F. A. Bais and P. Batenburg, Nucl. Phys. B 253 (1985) 162; D. N. Page and C. N. Pope, Class. Quanum Grav. 4 (1987) 213; A. M. Award and A. Chamblin, ibid. 19 (2002) 2051.

[5] C. N. Yang, J. Math. Phys. 19 (1978) 320.

[6] G. 't Hooft, Nucl. Phys. B79 (1974) 276; A. M. Polyakov, J. E. T. P. Lett. 20 (1974) 194.

[7] M. Minami, Prog. Theor. Phys. 62 (1979) 1128.

[8] E. B. Bogomolny, Sov. J. Nucl. Phys. 24 (1976); M. K. Prasad and C. M. Sommerfield, Phys. Rev. Lett. 35 (1975) 760.

[9] R. Auzzi, S. Bolognesi, J. Evslin, K. Konishi and H. Murayama, hep-th/0405070, F. A. Bais, hep-th/0407197.

[10] K. Pilch and A. N. Schellekens, Nucl. Phys. B 256 (1985) 109.

[11] I. I. Cotăescu and M. Visinescu, Symmetries and supersymmetries of the Dirac operators in curved spacetimes, in Progress in General Relativity and Quantum Cosmology Research (Nova Science, 2005, in press). 
[12] T. Yoneya, J. Math. Phys. 18 (1977) 1759.

[13] Y. M. Cho and P. O. G. Freund, Phys Rev. D 12 (1975) 1711; A. S. Schwarz, Commun. Math. Phys. 56 (1977) 79; P. Forgacs and N. S. Manton, ibid. 72 (1980) 15; A. V. Guiduk, Theor. Math. Phys. 44 (1980) 795; G. Chaohao and H. Hesheng, Commun. Math. Phys. 79 (1981) 75; M. Henneaux, J. Math. Phys. 23 (1982) 830.

[14] I. I. Cotăescu and M. Visinescu, Mod. Phys. Letters A 19 (2004) 1397.

[15] L. H. Ryder, J. Phys. A: Math. Gen. 13 (1980) 437.

[16] B. Thaller, The Dirac Equation (Springer Verlag, Berlin Heidelberg, 1992).

[17] G. Mackey, Induced Representations of Groups and Quantum Mechanics (Benjamin, New York, 1968).

[18] I. I. Cotăescu, J. Phys. A: Math. Gen. 33 (2000) 9177.

[19] T. T. Wu and C. N. Yang, Phys. Rev. D 12 (1975) 3845.

[20] J. F. Cornwell, Group Theory in Physics (Academic Press, London, 1984). 